\author{
UNIVERSIDADE FEDERAL DE SANTA MARIA \\ Reitor: Paulo Afonso Burmann \\ Vice-Reitor: Paulo Bayard Dias Gonçalves \\ CENTRO DE EDUCAÇÃO FÍSICA E DESPORTOS \\ Diretor: Luiz Osório Cruz Portela \\ Vice-Diretora: Maria Amélia Roth
}

\title{
Editor
}

Elenor Kunz

\section{Comissão Editorial}

Alexandro Andrade (Universidade do Estado de Santa Catarina)

Alexandre Fernandez Vaz (Universidade Federal de Santa Catarina)

Andressa Aita Ivo (Universidade Federal de Santa Maria)

Daniela Lopes Santos (Universidade Federal de Santa Maria)

Elizara Carolina Marin (Universidade Federal de Santa Maria)

Ivone Job (Universidade Federal do Rio Grande do Sul)

João Francisco Magno Ribas (Universidade Federal de Santa Maria)

Maria Cecília Camargo Gunther (Universidade Federal de Santa Maria)

Pere Lavega Burgués (Universidade de Lleida)

\section{Editores de área}

Andressa Aita Ivo (Universidade Federal de Santa Maria)

Daniela Lopes dos Santos (Universidade Federal de Santa Maria)

Elizara Carolina Marin (Universidade Federal de Santa Maria)

\section{Conselho Editorial}

Alexandre Fernandez Vaz (Universidade Federal de Santa Catarina)

Alexandro Andrade (Universidade do Estado de Santa Catarina)

Ana Márcia Silva (Universidade Federal de Goiás)

Celi Zulke Taffarel (Universidade Federal da Bahia) 
Edison de Jesus Manoel (Universidade de São Paulo)

Jorge Ricardo Saraví (Universidade de la Plata)

Lilian Teresa Buken Gobbi (Universidade Estadual Paulista)

Pere Lavega Burgués (Universidade de Lleida)

Reiner Hildebrandt Stramman (Technische Universität Braunschweig)

Silvana Vilodre Goellner (Universidade Federal do Rio Grande do Sul)

Vicente Molina Neto (Universidade Federal do Rio Grande do Sul)

\section{Equipe Técnica/Editoração}

Felipe Barroso de Castro (Universidade Federal de Santa Maria)

Daniele Jacobi Berleze (Universidade Federal de Santa Maria)

\section{Capa e Arte Final}

Marina Smidt Mainardi

Kinesis [recurso eletrônico] / Universidade Federal de Santa Maria. Centro de Educação Física e Desportos. Vol. 1, n. 1 (jan./jul. 1985)-. Santa Maria, 1985-

Disponível em: http://cascavel.ufsm.br/revistas/ojs-

2.2.2/index.php/kinesis/index

Continuação a partir de 1984 da Revista do Centro de educação Física e Desportos.

Semestral

e-ISSN 2316-5464

Vol. 33, n. 2 (jul./dez. 2015)

1. Educação física - Periódico. 2. Desporto. I. Universidade Federal de Santa Maria - UFSM. II. Centro de Educação Física e Desportos - CEFD.

Ficha catalográfica elaborada por Fernando Leipnitz - CRB-10/1958 